\title{
LOS PROFESORES DE INGLÉS (RE)CLAMANDO POR UN CAMBIO EN EL CONCEPTO DE FORMACIÓN INICIAL DE PROFESORES ${ }^{1}$
}

\author{
Jairo Enrique Castañeda \\ Trujillo \\ Jairo.castaneda@usco.edu.co \\ Bachelor of Arts in Spanish and \\ English), Magister in Education with \\ an emphasis in English Didactics, \\ Assistant professor at Universidad \\ Surcolombiana, Neiva, Huila, Master's \\ in Language Teaching professor at the \\ Universidad Pedagógica y Tecnológica \\ de Colombia, Tunja, Colombia.
}

Received: March 18, 2019

Accepted: May 12, 2019

How to cite this article:

Castañeda-Trujillo, J. (2019). (Re)clamando por un cambio en el concepto de formación inicial de profesores. Enletawa Journal, 12(1), 62-79.

\section{RESUMEN}

La formación inicial de profesores de inglés se nutre de muchas voces, también se tienen en cuenta postulados de la Pedagogía Crítica. Los docentes en formación, solicitan que esas voces sean escuchadas, pues se hace necesario conocer las experiencias pedagógicas desde su perspectiva de sus protagonistas. Este artículo, intenta rescatar las voces de los profesores de inglés en formación, a través del análisis de sus reflexiones escritas, durante el proceso de práctica pedagógica. Los principales hallazgos muestran que los profesores de inglés en formación claman por un cambio en los procedimientos y la forma de ver la práctica docente, así como también el rol principal que tienen los mentores para poder escuchar las voces de los profesores de inglés en formación. Se concluye, entonces, que una alternativa de pedagogía crítica abre el espacio para ser reflexivo, comprender el contexto propio y problematizarlo $\mathrm{y}$, posteriormente, lograr un cambio.

Palabras claves: pedagogía crítica, profesores de inglés en formación, práctica pedagógica, voz.

\footnotetext{
${ }^{1}$ Research report.
} 
El punto de partida: escuchar las voces de los profesores de inglés en formación

"El peor error que podemos cometer como futuros docentes es pensar que nuestra profesión es la misma que hace muchas décadas, estamos acostumbrados a obedecer a los demás sobre qué hacer y cómo hacerlo en las aulas" (Paola ${ }^{2}$, reflexión escrita, mayo 25 de 2018), estas son las palabras de una profesora en formación que reflexionan acerca de sus experiencias en la práctica pedagógica. Lo que ella expresó permite ver que los profesores de inglés en formación han estado pensando seriamente sobre su profesión a partir de lo vivido durante su práctica pedagógica. En palabras de McLaren (2002) esta interpretación y articulación de las experiencias diarias de los profesores en formación está siendo representada por lo que de ahora en adelante llamaremos "voces", las cuales están cargadas de significado dentro del entorno particular donde se dan.

Adicionalmente, Canagarajah (2004) menciona que es mediante estas voces por lo cual es posible resistir, modificar o negociar las grandes estructuras sociales, pero solo desde un nivel micro-social. Canagarajah también asegura que son estas grandes

\footnotetext{
${ }^{2}$ Los nombres han sido cambiados para proteger la
}

estructuras sociales, o las 'instituciones' en palabras de Foucault (1972), las que usualmente hacen oír sus voces a nivel macro-social, imponiéndose y posicionándose a sí mismas como las poseedoras de la única voz que deben ser tenida en cuenta. De esta forma, las voces provenientes del nivel micro-social son silenciadas, invisibilizadas y hasta anuladas como fuente de conocimiento.

A lo largo de este artículo, se pretende mostrar cómo estas voces, originadas a partir de las experiencias vividas en el proceso de formación inicial de profesores de inglés, son manifestaciones de resistencia y una solicitud de cambio frente a lo que se constituye como "el deber ser" y el "deber hacer" de un profesor de inglés. De la misma forma, también se quieren mostrar estas voces para que sean escuchadas, ya que se ha percibido que, por lo general, en la literatura especializada no les da el lugar que corresponde; estas solo han sido utilizadas como un medio para obtener información (Castañeda-Trujillo, 2018; Castañeda-Trujillo \& Aguirre Hernandez, 2018).

Para recopilar las voces de los profesores de inglés en formación, y asumiendo el rol de mentor de la práctica 
pedagógica, se invitó a un grupo de ocho profesores de inglés en formación, que hacen parte de un programa de Licenciatura en Educación Básica con énfasis en español, inglés y francés de una universidad privada en Bogotá, para que escribieran sobre sus experiencias, percepciones, sentimientos e ideas que surgieron como resultado de su paso por el programa de licenciatura en lenguas extranjeras. Pare este momento, los profesores en formación ya llevaban más de cuatro años estudiando en el programa de licenciatura y estaban por comenzar su vida profesional como profesores de inglés.

Como parte del plan de estudios de la licenciatura en mención, los profesores en formación deben cursar tres semestres de práctica pedagógica, los cuales se pueden desarrollar en diferentes niveles escolares: primaria, secundaria o educación no formal. Durante estos semestres de práctica pedagógica, los profesores en formación están acompañados por un docente de la universidad, quien hace las veces de mentor y quien debe estar pendiente del desempeño y necesidades educativas de los practicantes, en relación con la preparación de las actividades para las clases y por ende, el desarrollo de las mismas en el salón de clase.

Éstas experiencias en la práctica pedagógica fueron variadas, pues los docentes en formación vivieron esta experiencia de la siguiente manera: cuatro de ellos hicieron sus tres semestres de práctica en colegios públicos con niños de primaria; dos tuvieron que hacer sus dos primeros semestres de práctica en instituciones de educación superior y los dos restantes en instituciones de educación no formal, todos como profesores de inglés. La diferencia de los contextos, hace que las experiencias se diversifiquen aún más y que lo relatado en sus escritos reflexivos tenga valor relevante. En las siguientes secciones, se presentan algunas de las ideas que surgieron a partir de las reflexiones tratando de respetar el punto de vista de los profesores de inglés en formación.

\section{La educación desde los ojos de los} profesores de inglés en formación

Paola, que está en su último semestre de práctica pedagógica, expresa sus preocupaciones sobre el sistema educativo actual, dice:

Durante siglos, el sistema educativo ha sido el mismo. Los estudiantes asisten a clases; el maestro está frente a ellos dictando una lección sobre cualquier tema teórico; los estudiantes memorizan la lección y al final, tendrán un examen para ver si 
han aprendido o no. (Reflexión escrita, mayo 25 de 2018)

Ciertamente, lo que Paola está resaltando aquí tiene que ver con lo que Freire llamó "educación bancaria" (Freire, 2008). En esta visión de la educación, los profesores son los que saben todo, tienen el derecho de tomar cada decisión sobre el contenido, la evaluación y los procedimientos de la clase, por lo que son considerados como los dueños del conocimiento, los que pueden depositar conocimiento en los cerebros de los estudiantes. A su vez, los estudiantes son percibidos como receptores pasivos, incapaces de pensar, proponer o aportar nada relacionado con su proceso de aprendizaje; su única función es memorizar y repetir conocimientos producidos por otros y transmitidos por el profesor de una forma acrítica. Ramos (2019) añade que la formación de educadores es vista como un entrenamiento centrado en la disciplina y en cómo enseñar la disciplina; para esto se instrumentaliza el conocimiento y se difunden ciertas técnicas preestablecidas para mejorar los procesos de enseñanza.

Esta situación no es diferente en la formación de profesores de inglés. Paola continúa: "En el caso de las clases de idiomas, [los estudiantes] reciben los temas, las estructuras, la forma en que deben pronunciar las palabras y en qué situación pueden usar ciertos tiempos verbales" (reflexión escrita, mayo 25 de 2018). No hay ninguna posibilidad de hacer las cosas de manera diferente. El problema es, entonces, que esta transmisión de conocimiento es una acción acrítica, que convierte la educación en un espacio de opresión, discriminación y represión, reflejando el sistema social que rodea la escuela (Akbari, 2008; Aliakbari \& Fajari, 2011; Crookes \& Lehner, 1998). En el mismo sentido, Carlos afirma: "parece que la educación está condenada a continuar siendo lo que siempre ha sido durante décadas: un lugar donde hay que repetir de principio a fin" (reflexión escrita, mayo 25 de 2018).

Aparentemente, no hay mucha esperanza en los profesores de inglés en formación, el futuro es un poco borroso y, según ellos, el conocimiento recibido durante el pre-grado no es suficiente:

Cuando ingresamos a la universidad para estudiar este programa académico, muchos de nosotros pensamos que nuestro principal objetivo, una vez que nos graduamos, era cambiar la educación, porque estábamos conscientes de que algo andaba mal. Pero ahora que tenemos la oportunidad de enfrentar este 
'monstruo' no estamos usando las herramientas que se nos están dando. Tal vez no sabemos cómo sacar a los estudiantes del conductismo o están tan acostumbrados que no encontramos otra manera de enseñar. (Carlos, reflexión escrita, mayo 25 de 2018).

La idea de cambiar la educación, se ha transformado en adaptación al sistema, perpetuación y continuación de lo establecido durante tanto tiempo. Incluso Carlos llamó a la educación como un "monstruo", algo que es extremadamente fuerte y enorme, algo que es muy complicado de vencer; y él continúa:

Parece que para muchos estudiantes que se graduaron recientemente de la universidad todas las cosas que aprendieron no son útiles en la vida real. Ellos piensan que el propósito de la universidad es solo darte un diploma para trabajar como maestro. (Carlos, reflexión escrita).

Estas palabras permiten reflexionar sobre el papel de las universidades, los programas de licenciatura y, especialmente, de los profesores responsables de la formación profesional de los futuros docentes de lenguas materna y extranjera. Es tal cual como lo menciona Ramos (2019), la formación debe ser entendida desde lo dialógico, desde el desarrollo de potencialidades más bien que desde el entrenamiento en ciertas metodologías de enseñanza. Se pude percibir, entonces, que lo que se necesita es un cambio de actitud de parte de los agentes principales del proceso de formación, de los procesos de tutoría de la práctica docente, algo que beneficie a los profesores de inglés en formación a ver la educación de manera diferente.

Lorena sugiere un buen comienzo para el cambio, dice ella: "La implementación de un plan de estudios en la universidad puede proporcionar a los estudiantes la posibilidad de integrar sus conocimientos, ideas y percepciones de lo que es práctica pedagógica con lo que realmente se necesita para ser un maestro. "(reflexión escrita, mayo 25 de 2018). Este plan de estudios debe estar dirigido a comprender las implicaciones, no solo los procedimientos, de la educación. Por ejemplo, es necesario que los mentores fomenten una transformación de las relaciones de poder verticalmente constituidas que no permitan el desarrollo integral de las personas y que se convierta en un mecanismo de opresión. Esta transformación podría ser posible instruyendo a los profesores de inglés en formación sobre cómo funciona la opresión, 
cuáles son los medios de control de los opresores, y cómo operan en la educación y la sociedad (Aliakbari \& Fajari, 2011; Pennycook, 1990).

\section{Se necesita una nueva actitud hacia la enseñanza}

Si queremos cambiar el hecho de que nuestros estudiantes sean vistos como máquinas y simplemente como un número en la cadena productiva, nosotros, como futuros maestros, no podemos pretender enseñar de la misma manera que hace siglos. El mundo está cambiando todo el tiempo y mediante la educación podemos garantizar una vida mejor $\mathrm{y}$ un futuro mejor para las nuevas generaciones. (Paola, reflexiones escritas, mayo 25 de 2018)

De la anterior cita se colige que los profesores de inglés en formación tienen una perspectiva crítica sobre las posibilidades en la educación y en la enseñanza de idiomas. La solicitud sobre el cambio de actitud está estrechamente relacionada con la idea de dejar de percibir el trabajo de los docentes como una actividad técnica, por ejemplo recibir instrucciones sobre cómo usar una máquina, sin ocasión para pensar en otras opciones, al igual que los trabajadores de las fábricas (Quintero Polo \& Guerrero Nieto, 2013). En otras palabras, Paola está sugiriendo que los profesores de inglés sean vistos como sujetos sociales; capaces de analizar su contexto social e histórico; que pueden relacionarse con el otro desde la comprensión de las diferencias y no desde la diferencia misma; un docente que reflexiona sobre su quehacer y sobre la influencia que esto tiene en la vida de las personas que educa; que sean conscientes de la multiplicidad de perspectiva que existen dentro de su salón de clase y se aprovechen de esta para que sus estudiantes sean productores de conocimiento; que proveen un espacio de esperanza y libertad, permitiendo así la transformación de las realidades de los estudiantes (Ramos Holguin, 2019).

Lorena agrega algunas ideas más sobre la importancia de tomar conciencia sobre el impacto de ser maestro:

Me doy cuenta de que ser docente no es un trabajo simple, no es como ser ingeniero, arquitecto o abogado. $\mathrm{Si}$ bien todas estas profesiones tienen una gran importancia en el mundo profesional, no tienen el mismo impacto que la profesión docente en la sociedad (Reflexión escrita, mayo 25 de 2018) 
Esta visión de los docentes como

profesionales, debe estar dirigida a luchar por tener un espacio en el que los mentores y los profesores de inglés en formación puedan alzar la voz y expresarse sobre el hecho de haber sido ignorados, invisibilizados y silenciados durante mucho tiempo (Castañeda-Trujillo \& Aguirre Hernandez, 2018; Quintero Polo \& Guerrero Nieto, 2013).

Del mismo modo, Samacá Bohórquez (2012) afirma que los docentes en servicio deben pensar el aula como un espacio de comunicación en el cual todos los participantes intervienen activamente en la búsqueda de soluciones a diferentes temas relacionados, no solo con el aprendizaje de idiomas sino con la vida misma. Estas acciones podrían llevar a los profesores de inglés en formación a convertirse en intelectuales transformadores, que tiene que ver con pensar conectado a la acción, enfrentarse a pedagogías instrumentales y contribuir a la transformación de la educación como sociedad, opuesta totalmente a lo hecho por los técnicos pasivos (Giroux, 1990; Kumaravadivelu, 2006; Pennycook A., 1990).

Aunque la idea de un cambio es clara, Carlos plantea algunas preguntas que merecen consideración antes de pensar en la transformación; él dice:

Este concepto de lo que sé y en lo que quiero llegar a ser es el paso que no estamos teniendo en cuenta, ni las razones por las que solo estamos viendo la práctica de la enseñanza como un requisito, y no como un espacio donde tenemos la oportunidad de demostrar la forma en que nos vemos a nosotros mismos como maestros. (Reflexión escrita, mayo 25 de 2018)

Carlos menciona que es necesario reflexionar sobre qué tipo de maestros quieren ser en el futuro. Exige un proceso de práctica pedagógica más reflexivo en el que puedan recuperar el sentido de responsabilidad que un maestro necesita tener; prestar atención a los aspectos que afectan la enseñanza y el aprendizaje diferentes de los métodos o técnicas, como los contextos, las circunstancias y las personas involucradas en la intervención pedagógica (Samacá Bohórquez, 2012).

Carlos continúa:

La pregunta que tenemos que establecer ahora es qué estamos haciendo mal o en qué etapa del proceso debemos enfocarnos más. Este problema no es fácil de resolver porque es parte de la 
reflexión que los estudiantes deben hacer, no es parte del programa ni del sistema educativo. (Reflexión escrita, mayo 25 de 2018)

De estas palabras se deduce claramente que se necesita un alto nivel de conciencia para iniciar el proceso de transformación de la educación y desafiar el statu quo perpetuador de la opresión y las injusticias. Solo después de ser consciente de los sucesos y su causas, es posible pensar en mejorar las condiciones sociales de los docentes y los estudiantes (Akbari, 2008; Diaz-Greenberg \& Nevin, 2003). Por esa razón, es necesario interrogarse sobre la posición del docente hacia la raza, la clase social o el género; aspectos que están presentes en las aulas; y luego analizar hasta qué punto estos aspectos han sido invisibilizados, o las voces de quienes participan en la escena educativa han sido silenciadas o rechazadas (Akbari, 2008; Canagarajah, 2005; Castañeda-Trujillo, 2018; Pennycook, 1990).

Los programas de formación de profesores y los maestros deben asumir el aprendizaje de idiomas de una manera libertadora del "ser", promoviendo un discurso de esperanza que brinde la posibilidad de explorar formas de mejorar las condiciones sociales, humanizar la educación y empoderar a los estudiantes a la acción que los lleve a responsabilizarse de sus propias vidas (Aliakbari \& Fajari, 2011). Como afirma Juliana, "como profesora en formación, tengo la capacidad de contextualizar y proporcionar un sentido lógico a mi enseñanza" (reflexión escrita, mayo 25 de 2018), lo que se puede complementar con lo que Pennycook (2001) quiso decir con pedagogía como una actitud, es decir, una forma de hacer (Canagarajah, 2005), que requiere constante reflexión e introspección; Juliana reconoce que ellos, como los profesores de inglés en formación, pueden hacerlo:

Tener este tipo de espacios (la práctica pedagógica) también nos da la oportunidad de desarrollar nuestra autonomía y responsabilidad, descubriendo la relación entre la teoría que aprendimos durante la especialidad y la práctica que nos permite ser más que simplemente transmisor de información, pero contribuidores de transformación. (reflexión escrita, mayo 25 de 2018). Según Freire (citado en Aliakbari \& Faraji, 2011), hay tres niveles de conciencia (intransitiva, semi transitiva y crítica) que se establecen de acuerdo a dos criterios: primero, qué tan conscientes están las 
personas sobre los problemas de poder y sus propias condiciones de vida; y segundo, qué hacen las personas para cambiar estos aspectos. En el nivel intransitivo, las personas no intentan cambiar su situación; cualquier cambio que ocurra se debe al destino. En el segundo nivel de conciencia, semi intransitivo, las personas son conscientes de las injusticias y pueden hacer que suceda un cambio de una en una; sin embargo, consideran sus problemas como parte de sus vidas, algo normal, sin ninguna influencia o causa externa. El tercer nivel de conciencia solo es posible a través de una praxis y reflexiones colectivas sobre las circunstancias que los rodean; en este nivel de conciencia crítica, las personas pueden hacer conexiones entre sus condiciones de vida y el origen de las injusticias, y así, tomar medidas para cambiar tales circunstancias (Aliakbari \& Fajari, 2011). Los programas de formación de profesores requieren establecer acciones dentro de sus procesos curriculares que ayuden al profesor en formación a alcanzar el nivel crítico de concienciación. No se trataría simplemente de cambiar lo que está escrito dentro de los currículos, ni de cambiar la bibliografía, se trata de hacer una verdadera transformación en la forma de concebir la educación y la formación de maestros. Los profesores de inglés en formación deben identificar diferentes situaciones conflictivas dentro de los entornos educativos y sus causas. Luego, trabajar todos juntos para encontrar alternativas y formas de integración con los miembros de la escuela para disminuir las posibles causas de la injusticia y la opresión en la escuela. De esta manera, podrán tener una voz, y proporcionar una a aquellos que han sido silenciados debido a problemas de poder.

\section{Reflexionando sobre la enseñanza de idiomas desede las experiencias de los profesores de inglés en formación}

"Creo que uno de los mayores errores que cometemos cuando aprendemos, enseñamos o nos ponemos en contacto con otros idiomas es pensar estructuralmente" (Paola, reflexión escrita, mayo 25 de 2018). Como lo expresa esta profesora en formación, existe una idea difundida sobre la división del lenguaje en pequeños fragmentos mientras se enseña, incluso en los programas de formación de profesores de lenguas (Castañeda-Trujillo, 2018), asumido como el camino correcto para enseñar un idioma y reproducido en la práctica pedagógica. Paola dice que: 
Esta tendencia de ver el lenguaje como una estructura tiene que ver con el temor de no ser un buen usuario del lenguaje. Probablemente, decir qué dicen las cosas es incorrecto porque no podemos seguir una estructura, o probablemente, la persona con la que estamos hablando no va a entender por las mismas razones. Tus profesores y la forma en que aprendes el idioma te dan la idea de que el lenguaje que estás utilizando no es el correcto. (Reflexión escrita, mayo 25 de 2018)

Esta visión del lenguaje como una entidad monolítica que no puede ser modificada, suele ser enseñada a través de todo el pregrado, aceptada, y reproducida por los profesores de inglés en formación durante la práctica pedagógica, lo que representa una opresión a nivel de conocimiento y que se expresa en la homogeneidad y estandarización de los saberes y las prácticas (Castañeda-Trujillo, 2018). Pennycook (1990) mencionó que este hecho se debe a la idea de "desarrollo y mantenimiento de un estado nación" (p.305), la cual es una visión política del lenguaje que trata de mantener la dominación y la supremacía sobre aquellos que no pertenecen a la "nación" y rechaza la idea de tener múltiples variedades de inglés, por lo que la variedad de lenguas habladas en diferentes territorios no son tenidas en cuenta en las políticas lingüísticas y educativas, promoviéndose y reflejándose en los ámbitos económico, político, intelectual y social. Fuerzas que persiguen los intereses de las naciones occidentales (Pennycook, 1998; Phillipson, 1992).

Se necesita un cambio en la forma de ver la enseñanza, el aprendizaje y el "ser", tal como lo dice Alejandra: "el lenguaje debe verse como una forma de vida que abarca múltiples elementos y no como un proceso de memorización de contenidos y estructuras sin ningún sentido comunicativo. "(reflexión escrita, mayo 25 de 2018). De la misma manera, Julio señala que "el inglés está destinado a comunicarse para no aprender fórmulas teóricas para aprenderlo", y agrega que los profesores de inglés en formación "debería centrarse en la comunicación en lugar de en la corrección" (reflexión escrita, mayo 25 de 2018).

Ingrid asegura que ellos, como profesores de inglés en formación, "tienen en sus manos el desafío de cambiar las mentes de los estudiantes con relación al lenguaje; eliminando la idea que aprender un idioma es aprender estructuras, y el lenguaje es solo un sistema necesario adquirir para 
tener buenas oportunidades "(reflexión escrita, mayo 25 de 2018). Aquí, es evidente que Ingrid habla acerca del aspecto neoliberal que se ha extendido en las instituciones educativas (escuelas, universidades, institutos, etc.) y, en consecuencia, el imaginario colectivo que relaciona el aprender inglés con tener éxito, tanto académico como económico. Esta idea ha sido difundida por organizaciones internacionales con el objetivo de centralizar el poder en unas pocas naciones y posibilitar la formación de trabajadores (Levidow, 2005). Las consecuencias de esta visión neoliberal de la educación tienen su herramienta no solo para los alumnos, sino también para los docentes, que se han sentido presionados para obtener niveles más altos de competencia en inglés y ajustarse a los requisitos de las instituciones que venden enseñanza de idiomas como producto.

Ingrid agrega que uno de sus propósitos como maestra es "mostrar [a los alumnos] que el inglés es útil para interactuar con personas de otros países, mirar televisión, entender textos académicos, investigar, o simplemente usarlo en su la vida cotidiana " (Ingrid, reflexión escrita, mayo 25 de 2018). Estos pensamientos invitan a todos los profesores de inglés a considerar formas alternativas de ver la enseñanza, a pensar más allá de lo que se dice, que generalmente se convierten en "sentido común", es decir, formas inmodificables de enseñar y aprender que perpetúan la opresión en las escuelas (Kumashiro, 2009). El autor también asegura que "una manera de que la derecha haya tenido éxito en cambiar la política educativa es a través de mensajes estratégicos que enmarcan lo que el público en general considera 'sentido común' sobre cómo deberían ser las escuelas y cómo deberían reformarse las escuelas" (p. xxii). Laura da un ejemplo de opresión durante su práctica docente, ella dice:

"Mientras enseñaba, llegó una maestra al salón de clases, una maestra que no era la maestra principal que siempre estaba conmigo. Ella no me permitió dar mi clase, me dijo 'eres solo una maestra de pre-servicio', probablemente pensó que como todavía estoy en la universidad no sé cómo organizar una clase, pero le di muchas clases hasta ahora, y estaba totalmente preparado para dar la clase" (reflexión escrita, mayo 25 de 2018) Lo que Laura vivió es precisamente una 
forma de discriminación que no se puede permitir en nuestro contexto, no entre los docentes. Estos hechos generan desigualdades, violencia, discriminación, privaciones, etc., que producen opresión y aversión, "las escuelas son espacios donde el Otro es tratado de manera perjudicial" (Kumashiro, 2000, p. 26).

Para resistir la opresión, los profesores de inglés en formación y los maestros en servicio deben: "Crear un ambiente que abra la oportunidad de dialogar entre ellos; para acordar qué es lo mejor para que nuestros alumnos aprendan y cómo hacerlo. Invitar a nuestros estudiantes a interactuar con ellos y con el maestro, adquiriendo y luego produciendo. Esto solo es posible si utilizamos formas innovadoras de educación y el mundo" (Paula, reflexión escrita, mayo 25 de 2018) Como mencionó Canagarajah (2005), debemos proporcionar un entorno educativo y social más equitativo y ético, pero se requiere una actitud hacia el aula y la sociedad. Es como Julio menciona:

"El inglés se debe enseñar desde una chispa que solo puede existir en cada persona y en su propio estilo. Debe crearse una chispa entre la relación profesor-alumno en la que el profesor motiva y los alumnos a encontrar una pasión por seguir o una meta que alcanzar, de modo que esta chispa puede convertirse en fuego, sin importar si los alumnos necesitan inglés o no en su futuro, lo usarán mientras lo necesiten para alcanzar sus metas / sueños; una verdadera pasión es la verdadera motivación para enseñar y aprender también en el caso del inglés" (reflexión escrita, mayo 25 de 2018) Lo que Julio intenta explicar aquí, es que hay una obligación en cada maestro de proporcionar a los estudiantes la motivación que ellos necesitan, o tal vez, el empoderamiento para que se sientan capaces de tomar su propia decisión respecto a lo que les conviene o no.

\section{Matices de esperanza en ELPT}

Aunque muchas cosas pueden parecer negativas, los profesores de inglés en formación todavía tienen esperanza en la profesión que han elegido:

"Ahora, entiendo que la enseñanza puede verdaderamente transformar la vida de una persona y que tiene un impacto inconmensurable. Ahora, la verdadera pregunta es, ¿hasta dónde estamos 
dispuestos a llegar para hacer un cambio significativo en la forma en que vemos la enseñanza, el aprendizaje y las personas con las que trabajamos?" (Camilo, reflexión escrita)

Lo que Camilo ve es una posibilidad de transformar el aprendizaje de idiomas. Él destaca que nosotros, como maestros, transformamos vidas, por lo que es nuestra responsabilidad asumir ese desafío desde el comienzo de nuestras carreras. Se expresa a sí mismo a partir de su experiencia en práctica pedagógica y, como dice Miguel, es "uno de los entornos de aprendizaje más importantes para representar el desarrollo docente de los profesores en formación" (reflexión escrita, mayo 25 de 2018). La práctica pedagógica permite a los profesores de inglés en formación "descubrir, en un solo lugar, diferentes formas de ver la vida, diferentes formas de pensar y expresarse" (Miguel, reflexión escrita, mayo 25 de 2018). Incluso sus miedos y debilidades están expuestos durante la práctica pedagógica:

"Después de casi 5 años de estudiar para ser docente, todavía no me veo como profesor de idiomas, solo con la experiencia que tengo de la práctica docente. Siento que es una profesión difícil porque deberías enfrentar muchos desafíos comenzando con el contexto en el que vas a enseñar, también la gestión de esta población y la forma en que debes adaptar el idioma a sus necesidades específicas" (Paola, reflexión escrita, mayo 25 de 2018). Aunque Paola expresa sus inseguridades, también manifiesta que es consciente de que ser docente de idiomas no es una tarea fácil, reconoce que hay muchos desafíos y que algunos de ellos son difíciles de cumplir. Sin embargo, las posibilidades siempre están ahí, y con más preparación y mayor conciencia de lo que implica ser un docente, los profesores de inglés en formación se transformará en agente o, como lo mencionó Samacá Bohórquez (2012), "transformadores y generadores de cambio de la sociedad", capaz de tomar decisiones informadas y enfrentar cualquier desafío (Boyte, 2016).

Sin embargo, la transformación que se mencionó anteriormente no es solo una responsabilidad de los profesores de inglés en formación, los mentores también deben participar en este proceso, tal como Jorge expresa:

"Creo que la práctica pedagógica es necesario para consolidar los conceptos adquiridos a lo largo de 
los principales, conceptos que luego se convertirán en actitudes necesarias para el desarrollo de las clases.

Entonces, la calidad de la educación ofrecida a los profesores de preservicio se refleja en la forma de enseñar mientras se desarrollan las clases" (reflexión escrita, mayo 25 de 2018).

"Ser docente es un trabajo tan admirable, y creo que es uno de los trabajos más ingratos de la sociedad" (Paola, reflexión escrita, mayo 25 de 2018), pero también es una profesión que ofrece esperanza a muchas personas.

\section{Las voces en la práctica pedagógica}

Hasta ahora, se han tratado de combinar las voces desde tres ángulos diferentes: el primero, desde los profesores de inglés en formación, que tienen un rol activo durante la práctica pedagógica, y que tienen muchas ideas de sus experiencias en las aulas que podrían contribuir a la formación docente. La segunda voz proviene de algunos autores que han desarrollado diferentes puntos de vista con respecto a la pedagogía desde una perspectiva crítica. Y finalmente, se proyecta incluir la voz del autor del presente escrito, como la voz del mentor en práctica pedagógica.

\section{La razón principal que fomenta esta} idea de tener diferentes voces es el hecho de que, en la educación tradicional, las voces que generalmente se escuchan son las que pertenecen al gobierno, personal administrativo en la escuela y, a veces, los profesores, pero los profesores de inglés en formación han sido silenciados y, desde éste punto de vista, deben ser escuchados porque representan el futuro de la profesión (McLaren, 2002; Castañeda-Trujillo, 2018; Ramos Holguin, 2019). Escuchar las voces de los profesores de inglés en formación podría ayudar a los mentores y al personal administrativo a ser más receptivos y conscientes de las dificultades, formas de acercamiento a la educación, alternativas en metodologías e incluso diferentes aspectos culturales que pueden afectar la enseñanza y el aprendizaje de idiomas (Diaz-Greenberg \& Nevin, 2003).

En consecuencia, al proporcionar espacios donde estas voces pueden ser escuchadas y legitimadas, los profesores de inglés en formación pueden iniciar un proceso de liberación de la opresión causada por diferentes factores. Además, podría darles a los profesores de inglés en formación la posibilidad de ejercer el poder en su contexto local, y la posibilidad de explorar formas de cambio para una vida 
mejor y más democrática (Akbari, 2008).

No obstante, escuchar las voces los profesores de inglés en formación implica mucho más que solo escuchar lo que dicen; más bien, escuchar implica hacer posible la articulación de realidades a través del diálogo. De esta forma, podría ser posible obtener una comprensión más amplia de los valores, ideologías, principios y otros asuntos que los profesores de inglés en formación tiene con ellos y que pueden contribuir a mejorar la práctica pedagógica (McLaren, 2002; Pennycook, 2001). Pero lo más importante, es crear un ambiente en el cual cada uno de los participantes pudiera contribuir desde sus puntos de vista, generando con esto un sentido democrático de respeto por la diferencia que es esencial para cualquier noción de igualdad en la sociedad (Giroux citado en Pennycook, 1990), utilizando las experiencias vividas de los profesores de inglés en formación como narración de agencia y como referencia para la crítica.

\section{Conclusiones}

Después de analizar las voces de los profesores de inglés en formación, es posible notar que existen algunas manifestaciones de resistencia. Como afirma Méndez (2018) la resistencia surge cuando hay que enfrentarse a la dominación. Para el caso de los profesores de inglés, esta dominación surge principalmente del sistema educativo, el control que se quiere ejercer sobre el "ser" imponiendo una forma única de profesor, y del "hacer" formulando estrategias estandarizadas que no permiten el agenciamiento del profesor. Sin embargo, los profesores en formación se resisten a la idea de que exista una única forma de "ser" y de "hacer"; proponen alternativas direccionadas a comprender al otro y a ellos mismos como profesores. Se resisten a la idea de no ser libres, de estar atados a un sistema educativo que no escucha a los profesores ni mucho menos a los profesores en formación.

Esta resistencia implica una transformación del individuo y, de acuerdo con sus voces, también conlleva una esperanza para lo que será la educación en el futuro. Continuar pensando en la enseñanza del inglés como la transmisión de un cumulo de conocimiento lingüísticos y metalingüísticos debe detenerse. Los profesores de inglés en formación conocen las consecuencias de seguir perpetuando estas formas de hacer, y claman por una pedagogía abierta al dialogo, a la comprensión, al reconocimiento del otro; para esto es necesario que ellos mismos se 
arriesguen a pensar y a actuar diferentes, deben estar dispuestos a romper los esquemas y a superar los temores de ser encasillados como los rebeldes.

Los profesores de inglés en formación, tienen muchas cosas valiosas que decir y aportar, si queremos repensar la formación de profesores de inglés. Esas percepciones sobre la educación no pueden estar equivocadas; ellos han vivido y tratado con el sistema durante muchos años, y ahora están preocupados por ingresar a él y seguir siendo parte de la cadena productiva que perpetúa la injusticia y la opresión que han enfrentado. Sus experiencias son la manifestación de su agenciamiento a través de su propio proceso de formación. El escuchar estas voces debería ser un compromiso tanto de los profesores y directivos de los programas de formación de profesores de inglés. Se deben establecer acciones de mejora que propendan a lo dialógico, reflexivo e introspectivo, que abran la posibilidad de comprender mejor lo que pasa al interior de ellos mediante escuchar las voces de los profesores en formación.

\section{References}

Akbari, R. (2008). Transforming lives: introducing critical pedagogy into ELT classrooms. ELT Journal, 62(3), 276-283. doi:10.1093/elt/cen025

Aliakbari, M., \& Fajari, E. (2011). Basic principles in critical pedagogy. IPEDR, 17, 77-85.

Boyte, H. C. (2016). "A liberation of powers": Agency and education for democracy. Educational Theory, 66(1-2), 127-145.

Canagarajah, S. (2004). Multilingual writers and the struggle for voice in academic discourse. In A. Pavlenko, $\&$ A. Blackledge, Negotiation of Identities in Multilingual Contexts (pp. 266-289). United Kingdom: Multilingual Matter Ltd.

Canagarajah, S. (2005). Critical pedagogy in L2 learning and teaching. In E. Heinkel (Ed.), Handbook of research in second language teaching and learning (Vol. I, pp. 931-949). New Jersey: Lawrence Erlbaum Associates.

Castañeda-Trujillo, J. E. (2018). Voices from the south: English Language Pre-Service Teachers contributions to ELTE. In H. Castañeda-Peña, C. H. Guerrero, P. M. Rivera, A. C. Londoño, A. M. Rubio, C. A. Cepeda, ... Y. Samacá, ELT Local Research Agendas I (pp. 192-218). Bogotá: Universidad Distrital Francisco José de Caldas.

Castañeda-Trujillo, J. E., \& Aguirre Hernandez, A. J. (2018). Pre-service English teachers' voices about the teaching practicum. How Journal, 25(1), 156-173. 
Crookes, G., \& Lehner, A. (1998). Aspects of process in an ESL critical pedagogy teacher education course. TESOL Quarterly, 32(2), 319-328.

Diaz-Greenberg, R., \& Nevin, A. (2003). Listen to the voices of foreign language student teachers: Implications for foreign language educators. Language and Intercultural Communication, 3(3), 213-226.

Foucault, M. (1972). The archeology of knowledge. New York: Pantheon.

Freire, P. (2008). Pedagogy of the oppressed. New York: Continuum.

Giroux, H. (1990). Los profesores como intelectuales: hacia una pedagogia crítica. Barcelona: Paidos.

Kumaravadivelu, B. (2006). Understanding language teaching: From method to postmethod. Mahwah, New Jersey: Lawrence Erlbaum.

Kumashiro, K. (2000). Toward a theory of anti-oppressive education. Review of Educational Research, 70(1), 25-53.

Kumashiro, K. (2009). Against common sense: teaching and learning towards Social Justice. New York: Routledge.

Levidow, L. (2005). Neoliberal agendas for higher education. In A. Saad-Filo, D. Johnston, A. Saad-Filo, \& D. Johnston (Eds.), Neoliberalism: A critical reader (pp. 156-162). London: Pluto Press.

McLaren, P. (2002). Teachers and atudents. In P. McLaren (Ed.), Life in schools. An introduction to critical pedagogy in the foundations of education (pp.
242-253). Los Angeles: University of California.

McLaren, P. (2007). Life in schools: An introduction to critical pedagogy. Boston: Pearson Education.

Méndez, P. (2018). Schoolteachers' resisted images: Facing stereotypes and affirming identities. International Education Studies, 11(5), 100-109. doi:10.5539/ies.v11n5p100

Pennycook, A. (1990). Critical pedagogy and second language education. System, 18(3), 303-314.

Pennycook, A. (1990). Critical pedagogy and second language education. System, 18(3), 303-314.

Pennycook, A. (1998). English and the discourses of colonialism. London, New York: Routledge.

Pennycook, A. (2001). The politics of pedagogy. In A. Pennycook (Ed.), Critical Applied Linguistics: A Critical Introduction (pp. 114- 140). New Jersey: Lawrence Erlbaum Associates.

Pennycook, A. (2004). Critical moments in a TESOL praxicum. In B. Norton, \& K. Toohey (Eds.), Critical Pedagogies and Language Learning (pp. 327-346). Cambridge: Cambridge University Press.

Phillipson, R. (1992). Linguistic imperialism. New York: Oxford University Press.

Quintero Polo, A. H., \& Guerrero Nieto, C. H. (2013). "Of being and not being:" Colombian public elementary school teachers' oscillating identity. How Journal, 190-205. 
Ramos Holguin, B. (2019). Sentidos de la formación de educadores en idiomas modernos en la Universidad Pedagógica y Tecnológica de Colombia. Tunja, Boyaca: Editorial UPTC.

Samacá Bohórquez, Y. (2012). On rethinking our classroom: A critical pedagogy view. How Journal, 194208. 